\title{
Search for lepton flavour violation with the ATLAS experiment
}

\author{
B. $\mathrm{Le}^{1}$, on behalf of the ATLAS Collaboration
}

1 ARC Centre of Excellence for Particle Physics, The University of Melbourne

* brian.le@cern.ch

November 14, 2018

Proceedings for the 15th International Workshop on Tau Lepton Physics, Amsterdam, The Netherlands, 24-28 September 2018

scipost.org/SciPostPhysProc.Tau2018

\begin{abstract}
Lepton flavour violation (LFV) is a striking signature of potential beyond the Standard Model physics. The search for LFV with the ATLAS detector is reported in searches focusing on the decay of the Higgs boson, the $\mathrm{Z}$ boson and of a heavy neutral gauge boson, Z', using pp collisions data with a center of mass energy of $8 \mathrm{TeV}$ and $13 \mathrm{TeV}$.
\end{abstract}

\section{Contents}

\begin{tabular}{lll}
\hline & Introduction & 1
\end{tabular}

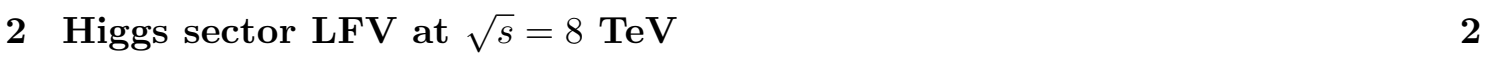

\begin{tabular}{|lll}
\hline 3 & LFV $Z$ boson decays & 4
\end{tabular}

3.1 Search using $\sqrt{s}=8 \mathrm{TeV}$ collisions 4

3.2 Search using $\sqrt{s}=13 \mathrm{TeV}$ collisions 4

\begin{tabular}{|lll}
4 & High mass resonance decays at $\sqrt{s}=13 \mathrm{TeV}$ & 6
\end{tabular}

\begin{tabular}{|lll}
5 & LFV in top quark decays at $\sqrt{s}=13 \mathrm{TeV}$ & $\mathbf{6}$
\end{tabular}

\begin{tabular}{lll|}
\hline 6 & Conclusion & 6
\end{tabular}

\begin{tabular}{ll}
\hline References & 7
\end{tabular}

\section{Introduction}

In the search for new physics phenomena at the LHC [1], searches for one class of models have begun to become rather sensitive with the increase in luminosity within the reach for new $\sqrt{s}=13 \mathrm{TeV}$ collisions. Charged lepton violation is predicted by a number of 
extensions to the Standard Model (SM) including models of supersymmetry (SUSY) [2 5], composite Higgs [6,7] and Randall-Sundrum models [8].

The search for charged lepton violation, which is normally the domain of low energy precision experiments, has reached the grasp of the high energy LHC. In particular, the measurements of specific couplings to neutral boson decays have become rather sensitive due to the quickly growing dataset. The focus of this summary is to give a brief overview of searches for charged lepton flavour violation performed with the ATLAS detector [9] with a specific focus on decays involving $\tau$ leptons.

\section{Higgs sector LFV at $\sqrt{s}=8 \mathrm{TeV}$}

One of the design objectives of the LHC was to measure the properties of the Higgs bosons in its numerous decays to SM particle pairs. Several extensions of the SM such as the two Higgs doublet (2HDM) 2 5], composite Higgs [6,7] and Randall-Sundrum models [8] can add additional charged lepton flavour violating (LFV) couplings to the Higgs sector. These LFV couplings can be probed with the ATLAS dector in decays of the Higgs boson to a pair of oppositely charged, different flavoured lepton pairs (i.e. using decays to $e^{ \pm} \mu^{\mp}$, $\mu^{ \pm} \tau^{\mp}$ and $e^{ \pm} \tau^{\mp}$ pairs).

The sensitivity to $Y_{e, \tau}$ and $Y_{\mu, \tau}$ non-diagonal Yukawa elements has been shown to be particularly large at the LHC through potential $H \rightarrow e \tau$ and $H \rightarrow \mu \tau$ decays. The current results from the ATLAS collaboration are from an analysis of the $\sqrt{s}=8 \mathrm{TeV}$ collisions in the search for $H \rightarrow e \tau$ and $H \rightarrow \mu \tau$ decays [10,11.

In the hadronic $\tau$ decay channel, the major backgrounds originate from $Z \rightarrow \tau \tau$ decays as well as from sources producing jets misidentified as hadronic $\tau$ candidates. The embedding method models $Z \rightarrow \tau \tau$ decays by taking $Z \rightarrow \mu \mu$ decays from data and replacing the muons with simulated $\tau$ decays to hadrons. The "OS-SS" method models the misidentified hadronic taus by taking a template from events in which the charge of lepton and hadronic $\tau$ candidates are the same. The normalisation of the template is taken from a multijet enriched region and is measured as the ratio between the number of events where the final states have opposite charge and the number of those which have same charge.

The signal is extracted from a maximum likelihood fit to the reconstructed mass calculated via Missing Mass calculator algorithm [12. The fit is performed in two regions which aim to reject either of the two major background components. . This is achieved by a $2 \mathrm{D}$ selection in the phase space of $m_{T}\left(e, E_{T}^{\text {miss }}\right)$ and $m_{T}\left(\mu, E_{T}^{\text {miss }}\right)$, one selection rejecting $Z \rightarrow \tau \tau$ and the other rejecting $W+$ jets events. The combination of both signal regions is presented in the plots in figure 1 .

The leptonic channel utilises a novel data-driven technique which exploits the (a)symmetry in event rates under the exchange of electron with muon between the BSM signal and SM backgrounds. As in the LFV decays the $p_{T}$ spectra of the lepton from the prompt decay of the Higgs boson is expected to be harder than that originating from the decay of the $\tau$ lepton (which is taken as a different flavoured lepton), events from the $H \rightarrow e \tau$ search can be modelled with data from $e \mu$ final states, wherein the $p_{T}(\mu)$ is harder than that of the $p_{T}(e)$. This strategy is also applied to the $H \rightarrow \mu \tau$ search. As the constraints on $\left|Y_{e, \mu}\right|$ are so strong [13], an LFV signature is only expected in one of the search channels (and not 

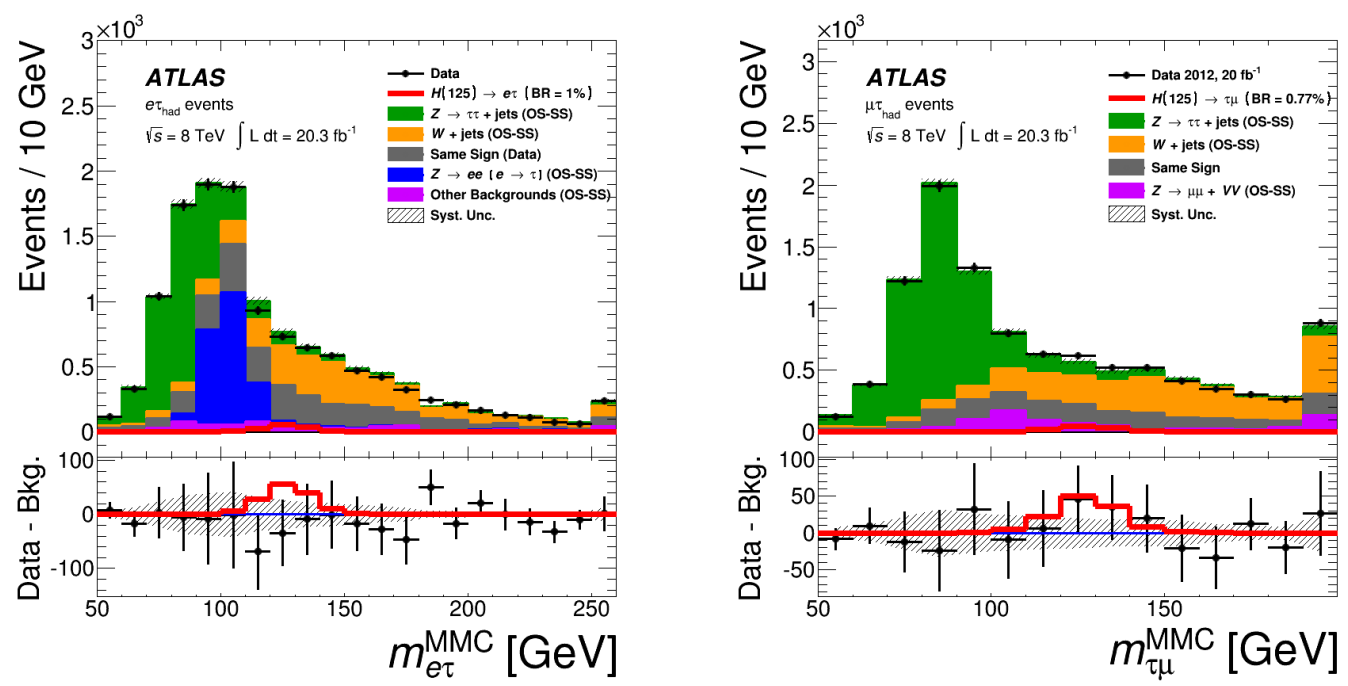

Figure 1: The distribution of the reconstructed di-tau mass in the $H \rightarrow e \tau$ (left) [11] and $H \rightarrow \mu \tau$ (right) 10 searches respectively. The lower panels represent the data/background agreement, with the red line representing the data/background+signal agreement.

the other). This asymmetry hypothesis is expected to be affected by experimental effects. These are accounted by modifying efficiencies, as well as in differences in the measurement of non-prompt leptons. The signal is extracted from a maximum likelihood fit to the Higgs boson mass, reconstructed using the collinear mass approximation, in two signal regions defined using the jet multiplicity. The distributions of the discriminating variables are presented in figure 2 .

In the combination of results, summarised in figure 3, one can see an excess only in
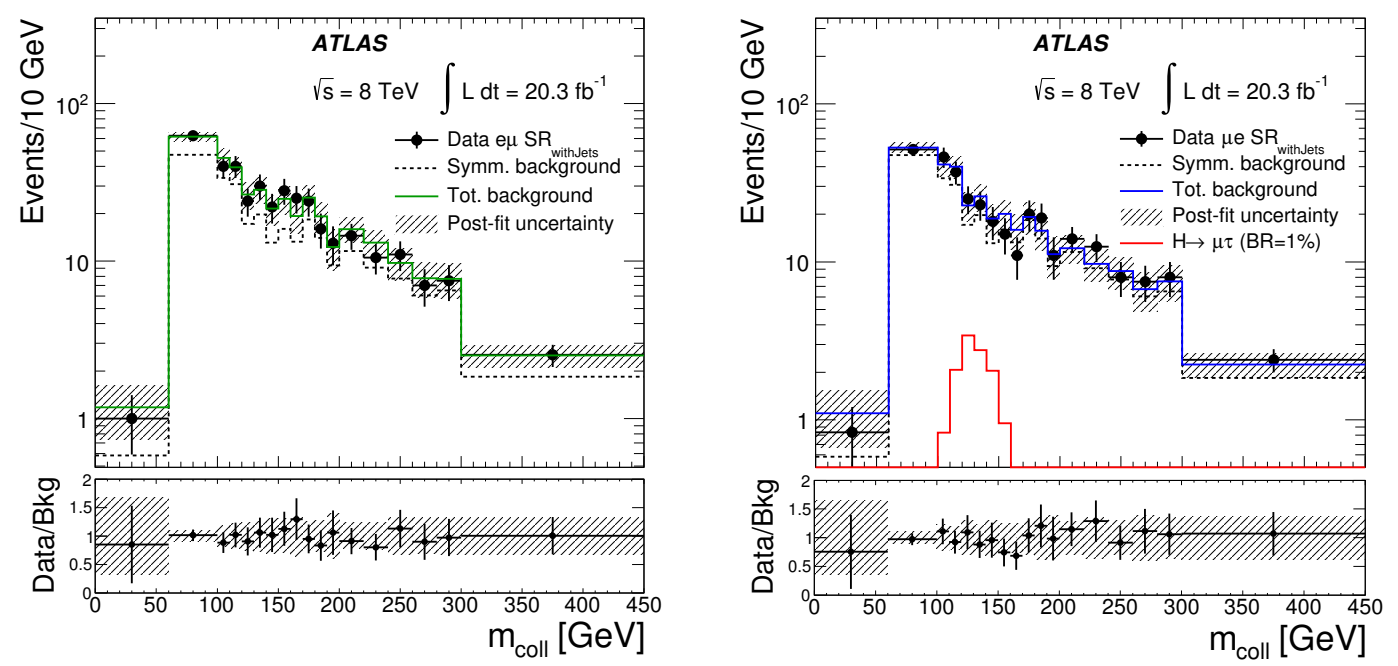

Figure 2: The distribution of the reconstructed di-tau mass in the $H \rightarrow e \tau$ (left) and $H \rightarrow \mu \tau$ (right) searches in the signal region with jets [11. The signal is shown only for the $H \rightarrow \mu \tau$ process. The lower panels quantify the agreement between data and background contributions.

the $H \rightarrow \mu \tau$ search in the hadronic channel which was found at a significance of $2.3 \sigma$. 
The upper limits on $H \rightarrow \mu \tau$ and $H \rightarrow e \tau$ branching ratios are set at $0.51_{-0.51}^{+0.51} \%$ and $-0.34_{-0.66}^{+0.64}$ with a best fit value of $1.43 \%$ and $1.04 \%$ respectively.

This search is expected to be updated with the $\sqrt{s}=13 \mathrm{TeV}$ collisions dataset where
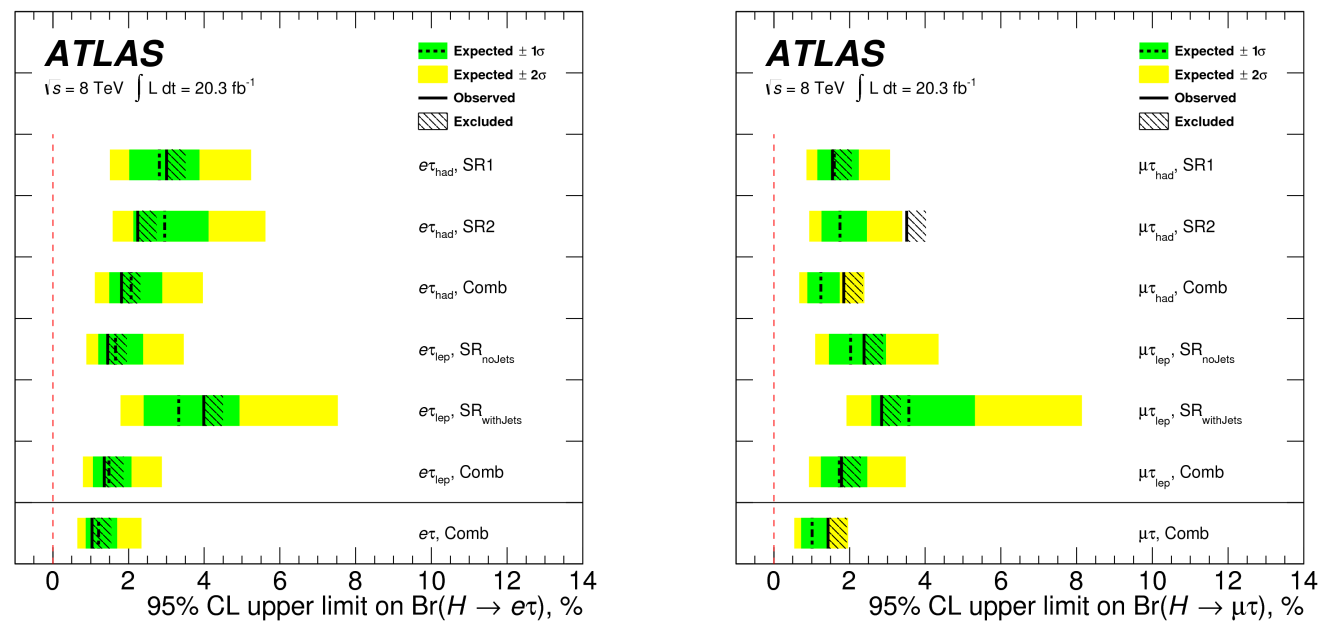

Figure 3: The expected and observed upper limits for branching ratios of $H \rightarrow e \tau$ (left) and $H \rightarrow \mu \tau$ (right) [11].

CMS has already provided the most stringent limits on the $\left|Y_{e, \tau}\right|$ and $\left|Y_{\mu, \tau}\right|$.

\section{$3 \quad$ LFV $Z$ boson decays}

\subsection{Search using $\sqrt{s}=8 \mathrm{TeV}$ collisions}

The presence of LFV decays can possibly be mediated by $Z$ boson couplings in heavy neutrino [14, extended gauge [15] and supersymmetry (SUSY) models [16]. A similar search strategy was employed for the search for LFV $Z \rightarrow \mu \tau$ decays in $\sqrt{s}=8 \mathrm{TeV}$ collisions [11. The thresholds on kinematic selections were modified to accommodate the lower mass of the neutral boson. Otherwise the overall search strategy remains consistent with that of the $H \rightarrow \mu \tau$ which was previously described. The distribution of the final discriminants in the two signal regions are illustrated in figure 4. No excess was observed and an upper limit on the branching ratio of this decay was set to $1.7 \times 10^{-5}$ with the best fit value consistent with no LFV coupling $-1.6_{-1.4}^{+1.3} \times 10^{-5}$.

\subsection{Search using $\sqrt{s}=13 \mathrm{TeV}$ collisions}

In the search using $\sqrt{s}=13 \mathrm{TeV}$ collisions, nearly all aspects of the measurement have been updated, including a new search for $Z \rightarrow e \tau$ decays 17]. Firstly, the background estimate was updated using a "fake factor". The OS-SS method events is limited in the sample used for the template region, defined by requiring the same charge between $Z$ decay products. The statistical power of the template can be improved by using events which fail the $\tau$ identification criteria rather than the charge criteria. These events are weighted with a different data-driven correction known as a "fake-factor" which depends on the rate of passing/failing the hadronic $\tau$ identification. This fake-factor is measured as a function of the $p_{T}$ of the hadronic $\tau$ candidate as well as the number of charged tracks. This allows 

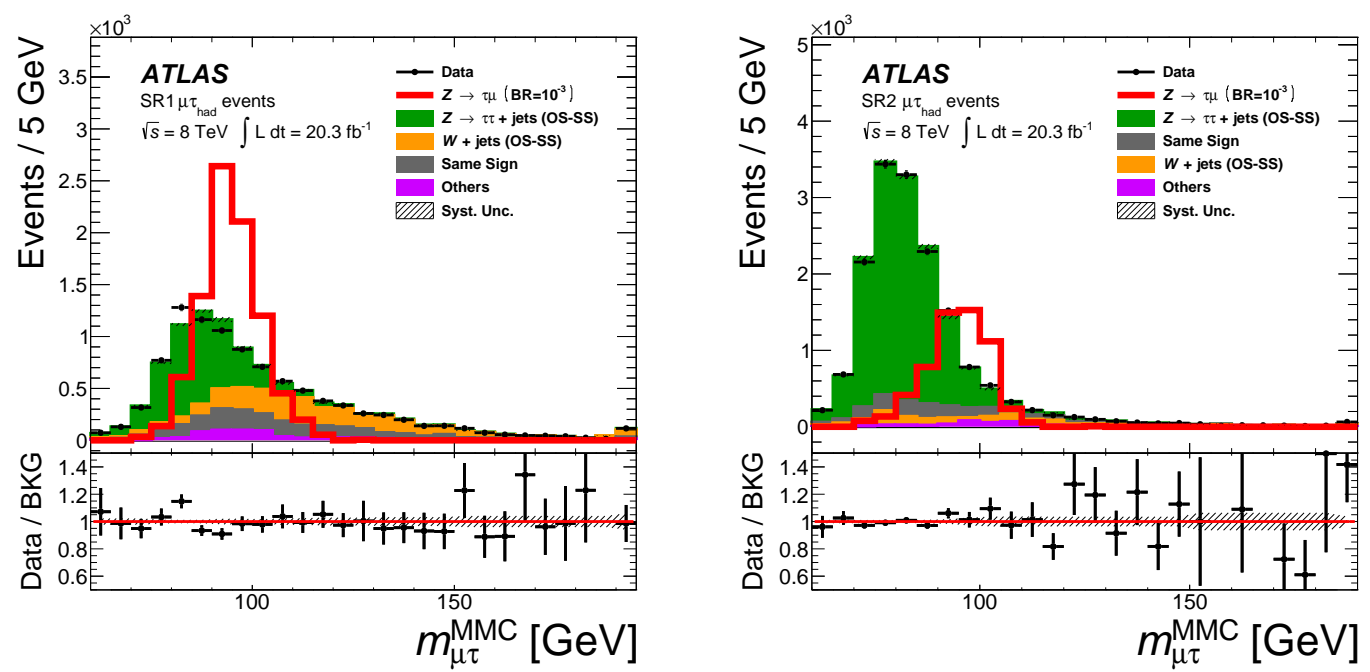

Figure 4: The distribution of the reconstructed di-tau mass in the search for $Z \rightarrow \mu \tau$ for the two signal regions which suppress the SM $Z \rightarrow \tau \tau$ (left) and $W+$ jets (right) backgrounds respectively [11. The lower panels represent the data/background agreement.

for a more accurate modelling of events from multijet and $W+$ jets processes in which a jet is misidentified as a hadronic $\tau$. In addition, the modelling of $Z \rightarrow \tau \tau$ candidates is now predominately Monte-Carlo driven, with a data-driven correction derived from within the fit.

The signal extraction has also been vastly improved, now relying on a multivariate approach involving neural networks. The neural networks are optimised separately depending on the number of charged tracks for each of the $Z \rightarrow e \tau$ and $Z \rightarrow \mu \tau$ searches. The networks are trained to classify the signal against one of the major backgrounds $(Z \rightarrow \tau \tau$, $W+$ jets and $Z \rightarrow l l)$. A total of 10 input variables are used for classification against $Z \rightarrow \tau \tau$ and $W+$ jets with an additional di-lepton mass variable used in training against $Z \rightarrow l l$ processes. The inputs include low level four-vector information for lepton, $\tau$ and $E_{T}^{m i s s}$ candidates, which are boosted, scaled and rotated for consistency, as well as higher level variables including $\Delta \alpha, p_{T}$ (total) and mass variables. The scores from the two or three networks are combined into a single classifier score and a binned maximum likelihood fit is used to extract the overall signal. The final discriminants are summarised in figure 5 .

The results for the $Z \rightarrow \mu \tau$ channel have been updated, still demonstrating no excess over the SM only hypothesis but allowing for an improved limit of $1.3 \times 10^{-5}$ in combination with the results from the $8 \mathrm{TeV}$ dataset. The new exclusion limit on $Z \rightarrow \mu \tau$ is competitive with LEP [18, which currently provides the most stringent bounds on such decays. A slight excess over the SM background has been observed in the $Z \rightarrow e \tau$ channel, not previously measured with ATLAS, of approximately $2.3 \sigma$. The best fit branching ratio for this search is $3.3_{-1.4}^{+1.5} \times 10^{-5}$. 


\section{High mass resonance decays at $\sqrt{s}=13 \mathrm{TeV}$}

In addition to the searches for known SM bosons decaying to LFV final states, a search for decays of non-SM high mass bosons in LFV final states is also performed [19,20. These probe three main models: $Z^{\prime}$ models with a single LFV vertex [21], R parity violating (RPV) SUSY models 22 and quantum black holes which are motivated by RandallSundrum and other extra dimensional models [23.

The major backgrounds for these searches originate from sources producing a misidentified hadronic $\tau$ candidate as well as from top quark decays. The $W+$ jets backgrounds are modelled from MC simulation with data-driven corrections for the misidentification probability of the hadronic $\tau$ candidate. The multijet background is modelled with a similar "OS-SS" approach previously described for the Higgs boson decays. The top background is extrapolated to the high mass signal region uing a fit performed on low mass simulated events.

The measurement was performed in $e \mu, \mu \tau$ and $e \tau$ decays. Results from $\mu \tau$ and $e \tau$ decays show no excess and allow for new constraints on the three main models motivating this search, as shown in figure 6. Interestingly, the constraints in the $Z^{\prime}$ and RPV-SUSY models are now stricter than those provided by low energy $\tau$ decay measurements as shown in figures 7 and 8 .

\section{$5 \quad$ LFV in top quark decays at $\sqrt{s}=13 \mathrm{TeV}$}

A new search has been performed to probe decays of top quarks to LFV final states [24]. This search is sensitive to dimension six operators which probe $t \rightarrow q e \mu$ (where $q=u, c$ ) decays.

The background modelling is performed using a matrix method, measuring the misidentification probabilities for the presence of non-prompt leptons. Signal extraction is performed using a fit to a boosted decision tree (BDT) classifier trained using 13 input variables.

No significant excess was observed, and the limits were set considering $\tau$ leptons couplings. These limits far exceed the limits set by low energy experiments. The overall distribution is shown in figure 9 .

\section{Conclusion}

The search for charged lepton violation using LHC collisions is a rich and varied area of research within ATLAS. Several searches are performed for decays of known SM bosons including the Higgs boson, which expected to produce new results for $\sqrt{s}=13 \mathrm{TeV}$ collisions. The latest results for $Z$ boson decays indicates a slight excess in the $Z \rightarrow e \tau$ channel and new improvedlimits for the $Z \rightarrow \mu \tau$ channel. High mass searches are now leading the sensitivity for LFV decays in $Z^{\prime}$ and RPV SUSY models. Finally LFV decays in the top quark sector are now providing new constraints to couplings which were previously unprobed. 
Copyright 2018 CERN for the benefit of the ATLAS Collaboration. Reproduction of this article or parts of it is allowed as specified in the CC-BY-4.0 license

\section{References}

[1] L. Evans and P. Bryant (editors), LHC Machine, JINST 3, S08001 (2008), doi:10.1088/1748-0221/3/08/S08001.

[2] J. D. Bjorken and S. Weinberg, A Mechanism for Nonconservation of Muon Number, Phys. Rev. Lett. 38, 622 (1977), doi:10.1103/PhysRevLett.38.622.

[3] J. L. Diaz-Cruz and J. J. Toscano, Lepton flavor violating decays of Higgs bosons beyond the standard model, Phys. Rev. D62, 116005 (2000), doi:10.1103/PhysRevD.62.116005, hep-ph/9910233

[4] M. Arana-Catania, E. Arganda and M. J. Herrero, Non-decoupling SUSY in LFV Higgs decays: a window to new physics at the LHC, JHEP 09, 160 (2013), doi:10.1007/JHEP10(2015)192, 10.1007/JHEP09(2013)160, [Erratum: JHEP10,192(2015)], 1304.3371.

[5] A. Arhrib, Y. Cheng and O. C. W. Kong, Comprehensive analysis on lepton flavor violating Higgs boson to $\mu^{\mp} \tau^{ \pm}$decay in supersymmetry without $R$ parity, Phys. Rev. D87(1), 015025 (2013), doi:10.1103/PhysRevD.87.015025, 1210.8241.

[6] K. Agashe and R. Contino, Composite Higgs-Mediated FCNC, Phys. Rev. D80, 075016 (2009), doi:10.1103/PhysRevD.80.075016, 0906.1542.

[7] A. Azatov, M. Toharia and L. Zhu, Higgs Mediated FCNC's in Warped Extra Dimensions, Phys. Rev. D80, 035016 (2009), doi:10.1103/PhysRevD.80.035016, 0906.1990.

[8] G. Perez and L. Randall, Natural Neutrino Masses and Mixings from Warped Geometry, JHEP 01, 077 (2009), doi:10.1088/1126-6708/2009/01/077, 0805.4652.

[9] ATLAS Collaboration, The ATLAS Experiment at the CERN Large Hadron Collider, JINST 3, S08003 (2008), doi $10.1088 / 1748-0221 / 3 / 08 /$ S08003.

[10] ATLAS Collaboration, Search for lepton-flavour-violating $H \rightarrow \mu \tau$ decays of the Higgs boson with the ATLAS detector, JHEP 11, 211 (2015), doi:10.1007/JHEP11(2015)211, 1508.03372.

[11] ATLAS Collaboration, Search for lepton-flavour-violating decays of the Higgs and $Z$ bosons with the ATLAS detector, Eur. Phys. J. C77(2), 70 (2017), doi:10.1140/epjc/s10052-017-4624-0, 1604.07730.

[12] A. Elagin, P. Murat, A. Pranko and A. Safonov, A New Mass Reconstruction Technique for Resonances Decaying to di-tau, Nucl. Instrum. Meth. A654, 481 (2011), doi:10.1016/j.nima.2011.07.009, 1012.4686

[13] A. M. Baldini et al., Search for the lepton flavour violating decay $\mu^{+} \rightarrow \mathrm{e}^{+} \gamma$ with the full dataset of the MEG experiment, Eur. Phys. J. C76(8), 434 (2016), doi:10.1140/epjc/s10052-016-4271-x, 1605.05081.

[14] J. I. Illana and T. Riemann, Charged lepton flavor violation from massive neutrinos in $Z$ decays, Phys. Rev. D63, 053004 (2001), doi:10.1103/PhysRevD.63.053004, hep-ph/0010193. 
[15] T.-K. Kuo and N. Nakagawa, Lepton Flavor Violating Decays of $Z^{0}$ and $\tau$, Phys. Rev. D32, 306 (1985), doi:10.1103/PhysRevD.32.306.

[16] F. Gabbiani, J. H. Kim and A. Masiero, $Z^{0} \rightarrow b \bar{s}$ and $Z^{0} \rightarrow \tau \bar{\mu}$ in SUSY: Are They Observable?, Phys. Lett. B214, 398 (1988), doi:10.1016/0370-2693(88)91384-6.

[17] ATLAS Collaboration, A search for lepton-flavor-violating decays of the $Z$ boson into a $\tau$-lepton and a light lepton with the ATLAS detector, Submitted to: Phys. Rev. (2018), 1804.09568.

[18] OPAL Collaboration, A search for lepton flavour violating z0 decays, Zeitschrift für Physik C Particles and Fields 67(4), 555 (1995), doi:10.1007/BF01553981.

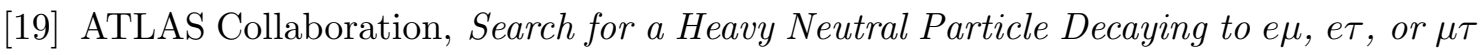
in pp Collisions at $\sqrt{s}=8 \mathrm{TeV}$ with the ATLAS Detector, Phys. Rev. Lett. 115(3), 031801 (2015), doi:10.1103/PhysRevLett.115.031801, 1503.04430.

[20] ATLAS Collaboration, Search for lepton-flavor violation in different-flavor, high-mass final states in pp collisions at $\sqrt{s}=13$ TeV with the ATLAS detector, Submitted to: Phys. Rev. (2018), 1807.06573.

[21] G. R. Farrar and P. Fayet, Phenomenology of the Production, Decay, and Detection of New Hadronic States Associated with Supersymmetry, Phys. Lett. 76B, 575 (1978), doi:10.1016/0370-2693(78)90858-4.

[22] R. Barbier et al., R-parity violating supersymmetry, Phys. Rept. 420, 1 (2005), doi:10.1016/j.physrep.2005.08.006, hep-ph/0406039.

[23] D. M. Gingrich, Quantum black holes with charge, colour, and spin at the LHC, J. Phys. G37, 105008 (2010), doi:10.1088/0954-3899/37/10/105008, 0912.0826.

[24] Search for charged lepton-flavour violation in top-quark decays at the LHC with the ATLAS detector, Tech. Rep. ATLAS-CONF-2018-044, CERN, Geneva (2018). 

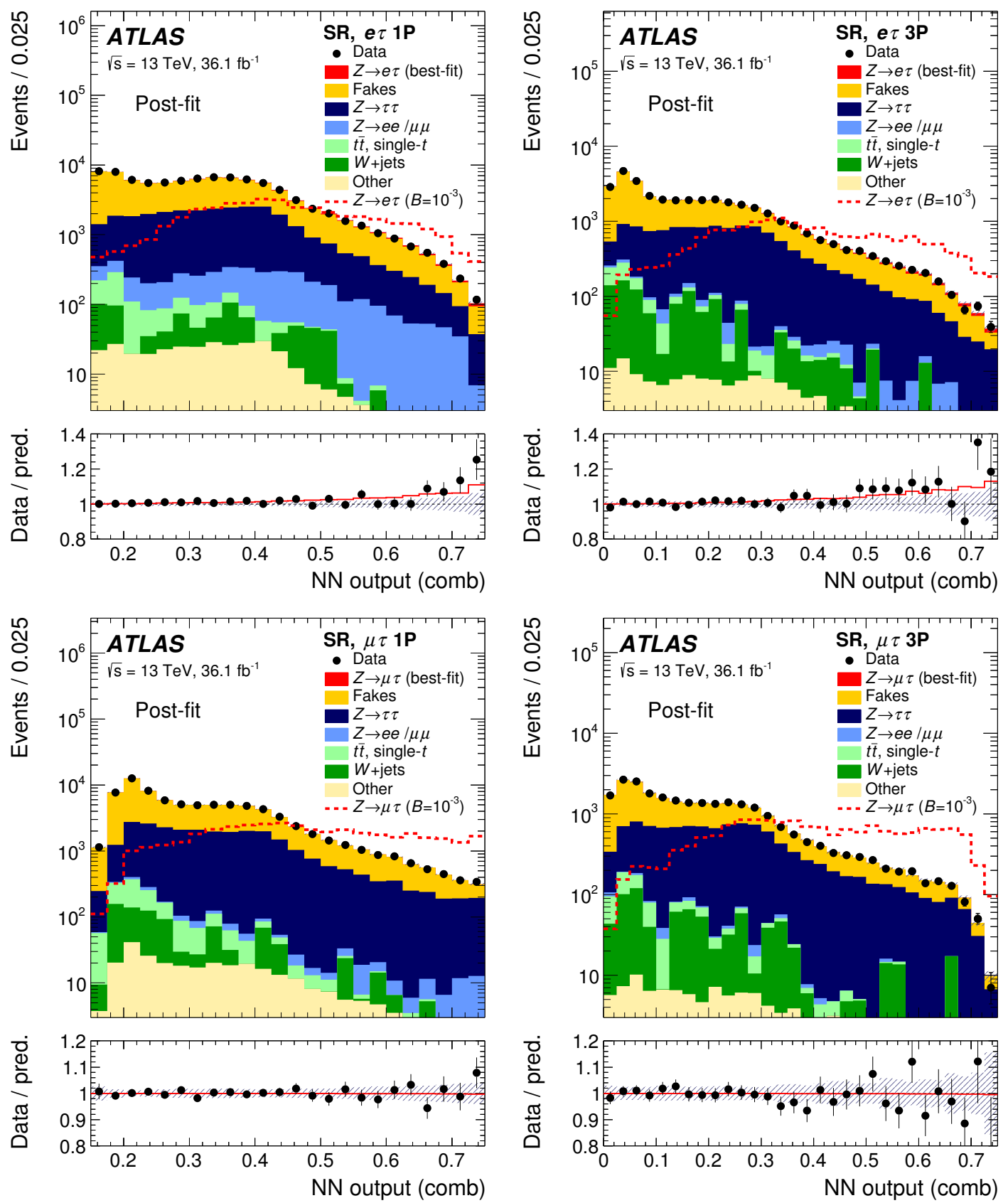

Figure 5: The distribution of the Neural Network classifier score for the $Z \rightarrow e \tau$ (top) and $Z \rightarrow \mu \tau$ (bottom) searches divided between the $1 \mathrm{p}$ and $3 \mathrm{p}$ decays respectively [17]. The lower panels represent the data/background agreement. For the $Z \rightarrow e \tau$, the signal fixed to the best-fit branching ratio plus background is represented on this plot as a red line. 

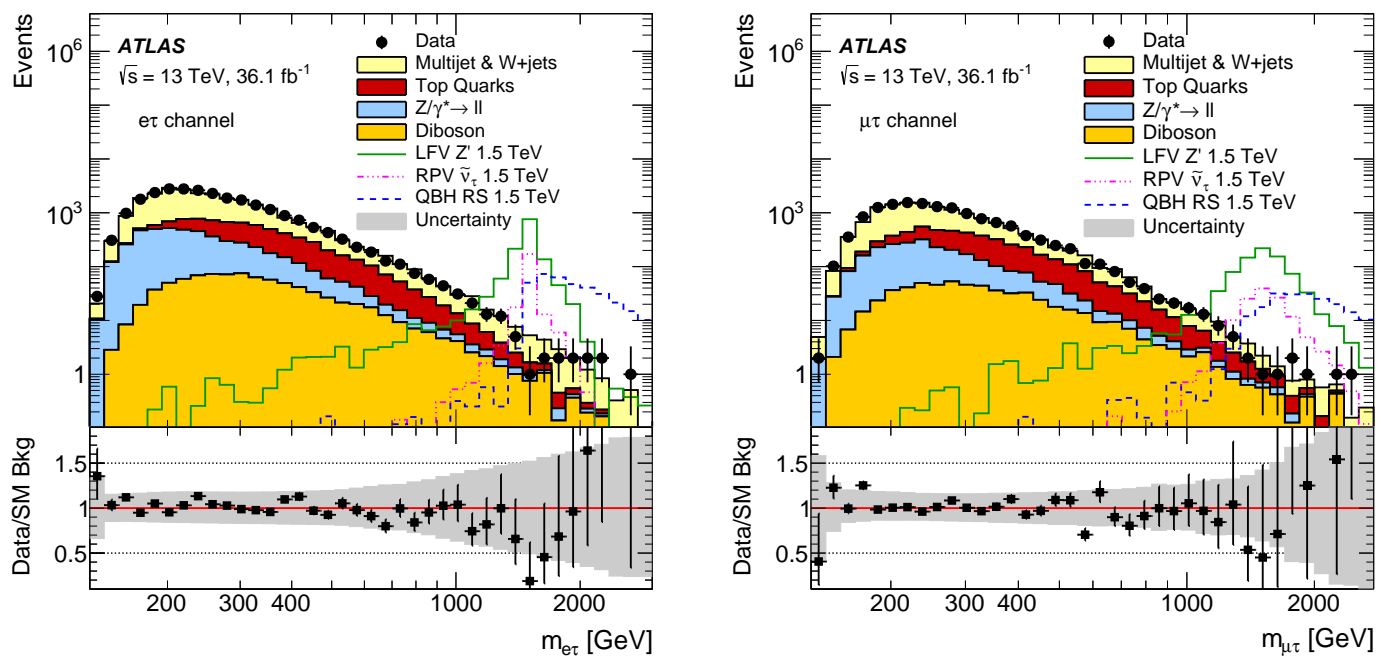

Figure 6: The final di-lepton mass distributions for $e \tau$ and $\mu \tau$ searches [20]. The various signal models to generate LFV are represented by unfilled, unstacked histograms.
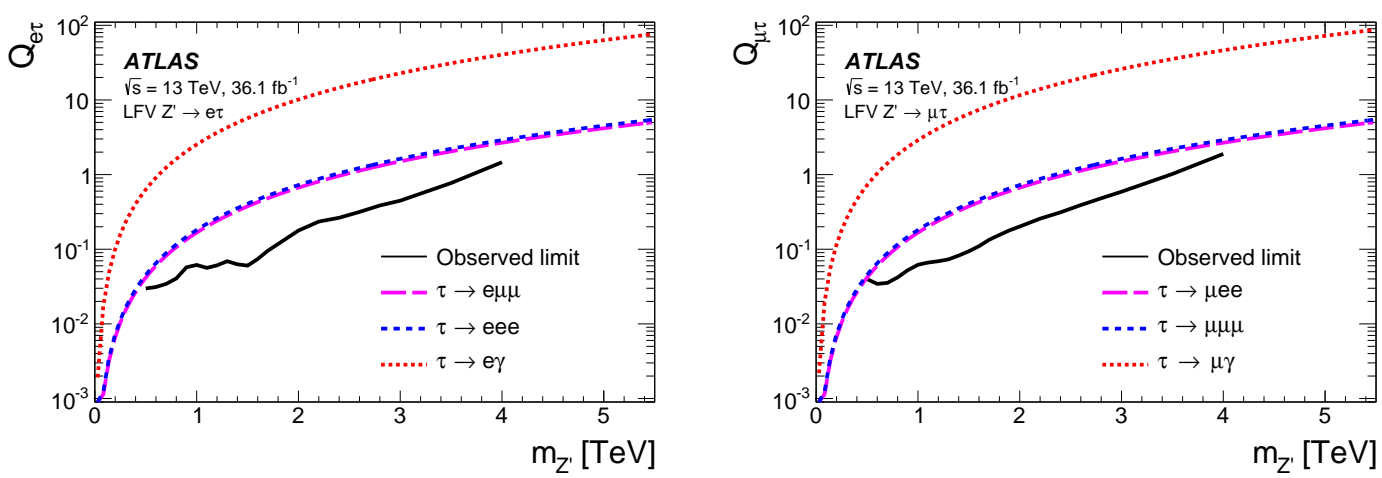

Figure 7: The observed upper limits on the lepton flavour violating $e \tau$ and $\mu \tau$ couplings as a function of a potential $Z^{\prime}$ boson 20 . The observed limit for $13 \mathrm{TeV}$ collisions now exceeds that of measurement of LFV via direct $\tau$ decay.
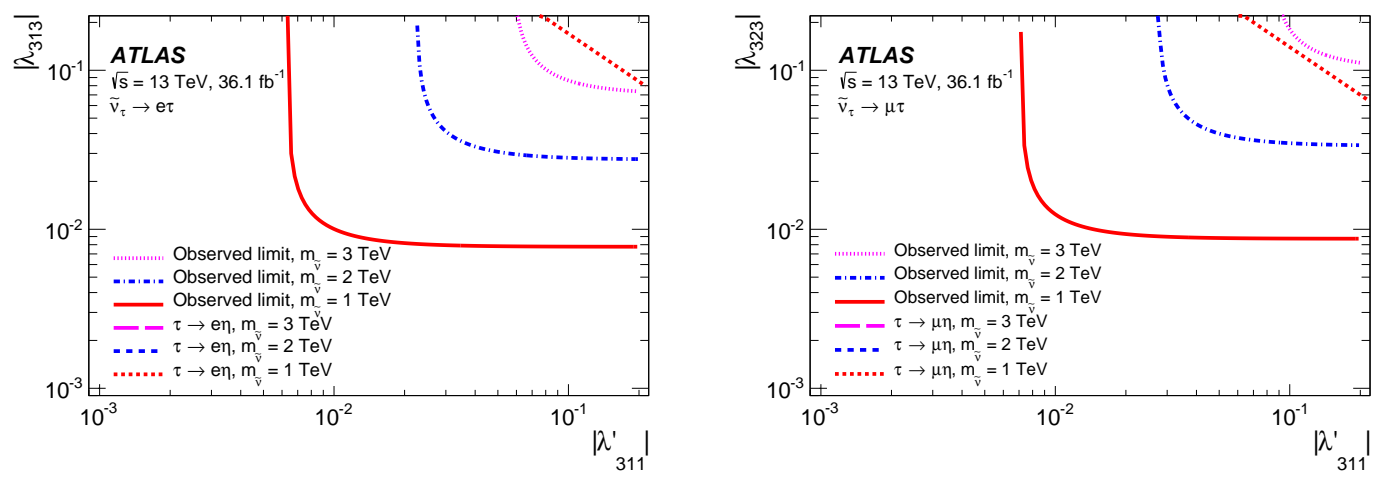

Figure 8: The observed upper limits on the lepton flavour violating $e \tau$ and $\mu \tau$ couplings as a function of another RPV coupling $\left|\lambda_{311}^{\prime}\right|$ (a coupling between first generation quarks and the tau-sneutrino) for a tau-sneutrino decay [20]. The observed limit for $13 \mathrm{TeV}$ collisions now exceeds that of measurement of LFV via direct $\tau$ decay. 

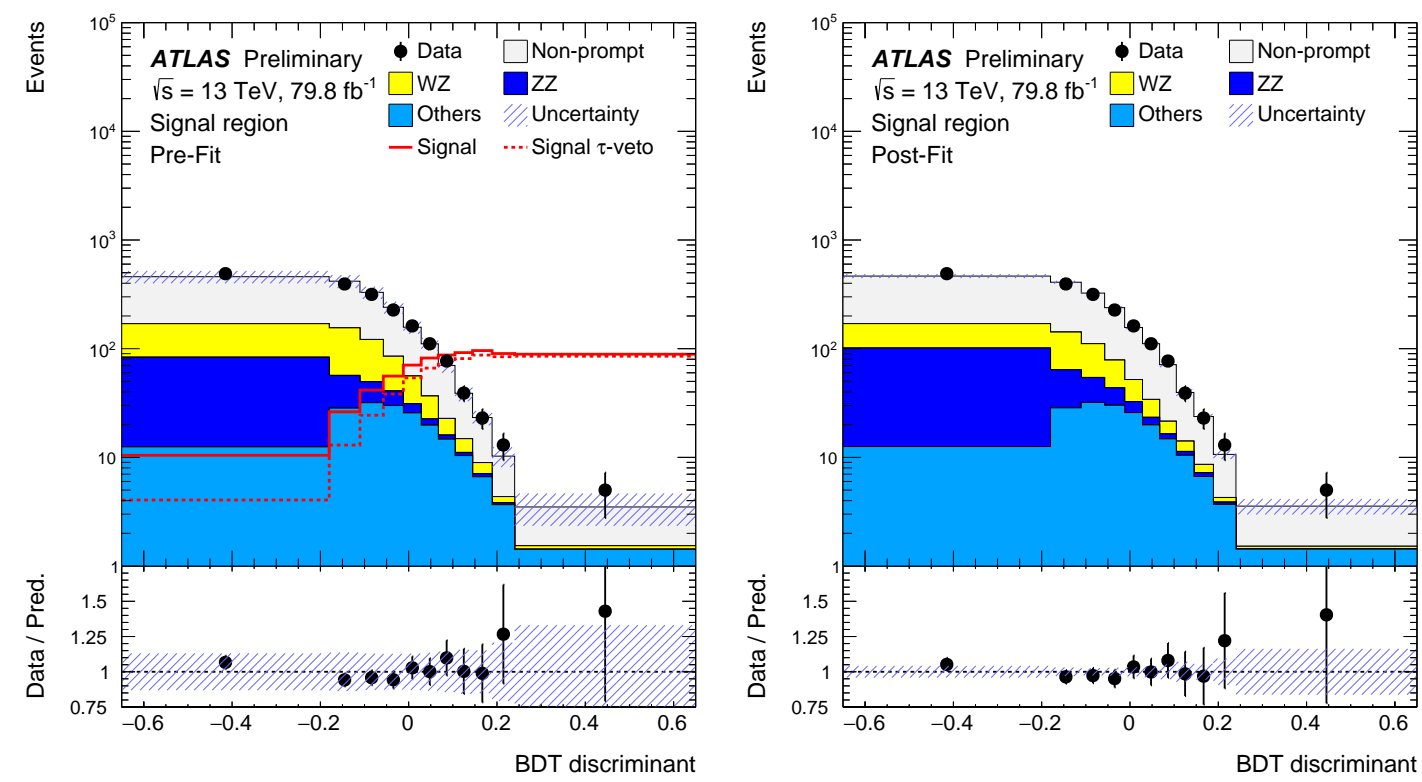

Figure 9: The pre-fit (left) and post-fit (right) distributions of the BDT classifier score 24. On the left plot, the signal is represented by the red histogram plotted unstacked. 